\title{
Alzheimer's disease care and management: Role of information technology
}

\author{
Hanuman Thota ${ }^{1}$, Allam Appa Rao ${ }^{2}$, Kiran Kumar Reddi ${ }^{1}$, Sivaprasad Akula ${ }^{1}$, Suresh Babu Changalasetty ${ }^{1}$, \\ Gedela Srinubabu ${ }^{2 *}$ \\ ${ }^{1}$ Department of Computer sciences and Engineering, Acharya Nagarjuna University, Guntur-522510, India; ${ }^{2}$ International Center for \\ Bioinformatics and Center for Biotechnology, Andhra University College of Engineering, Visakhapatnam-530003, India; \\ Gedela Srinubabu* - E-mail: srinubabuau6@gmail.com; * Corresponding author
}

received July 31, 2007; revised September 28, 2007; accepted October 19, 2007; published online November 03, 2007

\begin{abstract}
:
Alzheimer's disease (AD) an ailment that is supposed to affect people in old age. There are evidences that it might affect others also. The number of elders is increasing as the average life expectancy is increasing. AD afflicts its patients with the dementia and $\mathrm{AD}$ might increase in malignance over time. People with cognitive disabilities can be overwhelmed through cognitive prosthetics. With the help of information technology we can enhance the quality of life. Significant achievements are possible with an interdisciplinary approach that includes genomic, genetic, technological and therapeutic measures. The combination and coordination of Bioinformatics facilitates generation of various diagnostic tools for the people who are suffering from Alzheimer's disease. These tools help the care providers also. In this article, we emphasize the literature regarding the use of technology and its methodologies to improve the quality of care for the people with Alzheimer's disease.
\end{abstract}

Key words: information technology; bioinformatics; alzheimer's disease; electronic medical records

\section{Background:}

Alzheimer's disease (AD) was first discovered in 1907 by German neurologist Alois Alzheimer. [1] Alzheimer's disease becomes one of the major hurdles for further survival of elders thereby a many number of people may suffer from AD in the next few decades. Molecular genetics reached human genetics about 1976, when the first human genes were cloned [2] Transgenic methods, 'knock-outs' and 'knock-ins' began in about 1986, and in about 1996, database searching became a fruitful way to do genomic research [3] The term 'genome' refers to an organism's complete set of genes and chromosomes. The term genomics describes the scientific discipline of mapping, sequencing, and analyzing genomes. [4]

The fact that most diseases do not follow a simple inheritance patterns has led to a significant challenge in the genetic dissection of the complex traits of diseases such hypertension, Alzheimer's disease, schizophrenia and diabetes. Alzheimer's (AD) afflicts its patients with a dementia that increases in malignance over time: the older an $\mathrm{AD}$ patient is, the worse the dementia is. Dementia is a result of the loss of neurons in the brain that assist in engagement of intellectual activities. The loss of neurons specifically affects the hippocampus, which is a central region for memory operation, and the cerebral cortex. The cerebral cortex is also involved in memory functions, but also works to accomplish reasoning and language functions. A big difference between a normal brain and a brain afflicted with $\mathrm{AD}$ is the presence of protein clusters ISSN 0973-2063

Bioinformation 2(3): 91-95 (2007) inside and between neurons. The clusters inside are known as neurofibrillary tangles, which consist of a protein named tau. Another type of protein known as beta-amyloid is the protein that exists between neurons. While the presence of tau seems to be proportionate to the degree of dementia experienced by the patient, indicating a possible connection to the cause of $\mathrm{AD}$, it is not unique to $\mathrm{AD}$ the way betaamyloid plaques are in their unique concentrations. These beta amyloid proteins that cluster between neurons are accompanied by the immune system's microglia, reactive inflammatory cells that are thought to remove already damaged neurons and/or the amyloid plaques themselves. [1] They originate from the beta-amyloid precursor protein (bAPP) when a bAPP fragment that is 99 amino acids is cut by gamma-secretase, creating a beta-amyloid peptide, while the amyloid plaques themselves are present in most old people, the high concentration of them in the hippocampus and cerebral cortex in $\mathrm{AD}$ patients suggests a role in the neuronal degenerative process.

Memory loss is the most common and well known symptom for Alzheimer's disease. Other symptoms include loss of cognitive abilities, judgment, thinking and disorientation to place and time. Identification loss, depression, confusion, anxiety, fear, frustration, paranoia are also symptoms for Alzheimer's disease. The above symptoms may have different effects on different people. Currently medicines that are available for Alzheimer's disease slow down its progression or help control the 


\section{Bioinformation}

www.bioinformation.net

\section{Views \& Challenges}

symptoms such as anxiety or sleeplessness. However, there is no available cure for Alzheimer's disease. While the curative approach is certainly crucial to combating the effects of $\mathrm{AD}$, one avenue we might consider looking down is a focus on supplemental measures.

The development of new technologies that could help AD patients cope with loss of mental function might be appropriate, given the nature of the ailment. Developments in information technology could be offer assistance to $\mathrm{AD}$ patients in way that could supplement the loss of biological function with mechanical functions. For example, a computer could be used to keep records of family members to help remind the patient about his or her past. While a desktop PC seems somewhat impractical for this, a computer small enough to fit into someone's eyeglasses, coupled with voice and image recognition technology, could provide $\mathrm{AD}$ patients with the kinds of information they need to continue to function. This, along with drugs to at least slow the process, could provide a treatment that could restore a quality of life to the patient in a way that is currently unavailable.

A substantial number of the IMI patients reflected regional hypometabolism similar to $\mathrm{AD}$, suggesting that IMI is likely an early stage in progressive dementia. A large percentage of IMI patients converted clinically to AD within three years of initial study, though they observed impaired memory functioning well before a clinical diagnosis of $\mathrm{AD}$ could be made. In addition to potential clinical utility, IMI and PET represent an opportunity to study dementia in relation to brain chemistry at a time when brain pathology is in the process of development.

\section{Genomics of alzheimer's disease}

Genome analysis may be divided into structural and functional genomics. Structural genomics is an initial phase of genome analysis, and has a clear end point which is the construction of high-resolution genetic, physical, and transcript maps of an organism (its complete DNA sequence). This genotypic approach focuses on understanding how genotypic variation gives rise to phenotypic variation, relying on physical and genetic maps and easily-typed DNA sequence polymorphisms. The expression approach (functional genomics) relies on the large collection of partially sequenced cDNA clones. The benefits of the information arising from the accumulation of human gene sequences includes developing systematic ways of finding genes of interest, and their functions; hence 'functional genomics'. The genes cloned and their corresponding DNA sequences provide the tools for comprehensive characterization of the expression patterns of this entire set of genes, and for systematic experimental investigations of the functional properties of their products. Thus, functional genomics, which represents a new phase of genome analysis, makes use of the structural genomics information. The investigation is primarily a systematic approach to elucidate the genome and its functions.

ISSN 0973-2063

Bioinformation 2(3): 91-95 (2007)
Pathologically, Alzheimer's disease (AD) is associated with generalized degeneration of the cerebral cortical and hippocampal neurons. Cholinergic neurons in the basal forebrain which project to cortex and hippocampus appear to be particularly vulnerable, and to an extent, so are serotonergic and noradrenergic afferents to cortical regions. The extracellular deposition of peptide fragments (amyloidbeta) from the larger membrane precursor protein (APP) is typical in affected brain tissue. Intracellular accumulations of tau-proteins (tangles) are present in many cortical and cortico-limbic regions. [5]

Genetic studies have led to the identification of three genes in which mutations can cause $\mathrm{AD}$ : the $\beta$-amyloid precursor protein gene located on chromosome 21, presenilin 1 (PS1) located on chromosome 14 and presenilin 2 (PS2) located on chromosome $1 .[6,7,8]$ In addition, the E4 allele of the apolipoprotein E (ApoE) gene is a risk factor for AD. While mutations associated with APP are extremely rare, the 50 or so mutations associated with PS1 may explain up to half of all cases of early-onset AD. A study which investigated the association of two candidate genes (PS1 and $\alpha_{1}$ antichymotrypsin (ACT)) with the risk of sporadic Alzheimer's disease on chromosome 14 reported that the frequency of the $\mathrm{ACT}^{*} \mathrm{~A}$ allele was significantly higher in $\mathrm{AD}$ patients than in controls and the stratification of the ACT data by PS1 genotypes showed that the risk associated with the $\mathrm{ACT}^{*} \mathrm{~A}$ allele was confined to $\mathrm{PS} 1 * 1$ carriers only. [9]

The two-site haplotype data for PS1 and ACT indicated that the A1 haplotype, carrying the ACT*A and PS1 alleles, was more frequent in Alzheimer's disease patients, and these results may also suggest that there is a possible synergistic effect of these two loci on the risk of AD. In contrast to early-onset $\mathrm{AD}$, there is to date only one genetic factor indisputably linked with late-onset forms of this disorder; the E4 allele of apolipoprotein E. [10] Differences in ApoE genotyping appear to explain differences in patients' responses to drug therapy. With tacrine, a better response was seen in patients with the ApoE E2 or ApoE E3 allele than in those carrying the ApoE E4. The ApoE E4 allele has an inverse relationship with residual brain choline acetyltransferase (the acetyl-choline synthesizing enzyme) activity, and it appears that patients with this genotype may not have sufficient acetylcholine to benefit from a drug which acts as an inhibitor of acetylcholinesterase. However, patients with the ApoE E4 genotype appear to have a better response than other $\mathrm{AD}$ patients to treatment with another drug, Servier's S12024 (morpholinyl-2 methoxyl-8 tetrahydro-1, 2, 3, 4 quinoline) which is currently in phase II clinical trials. In fact, this drug had no detectable effect in patients with the other ApoE genotypes. S12024 does not appear to affect the cholinergic system, but rather to facilitate brain noradrenergic and vasopressinergic activity, and increases vasopressin synthesis and release in a dosedependent manner. There may be a balance between cholinomimetic and vasopressinergic pathways, according to ApoE E4 allele presence or absence. [11] With relevance 


\section{Bioinformation}

www.bioinformation.net

\section{Views \& Challenges}

to design of clinical trials, the important observation may be that alleles that appear to be conclusively associated with a therapeutically relevant phenotype can be used to select a subgroup of patients for clinical trials.

A polymorphic site need not be part of the target for the drug; it only needs to be associated with a response to the treatment. In responsive patients, the selective treatment could be more effective, and associated with fewer or less severe side effects. Furthermore, pre-emptive genotyping aimed at drug-associated genes could mean that fewer drug candidates would fail to reach the market place because of poor toxicity/efficacy profiles in the general population. For example, genotyping of early-onset AD is likely to include the two PS1 and PS2 genes involved in this disease. Predictive and diagnostic tests for PS1 mutations and diagnostic tests for ApoE alleles are already commercially available and other tests are being developed. Thus, genetic testing for $\mathrm{AD}$ exists for clinical use, and is likely to be used more often to stratify patients in Alzheimer's disease research, both in trials of preventive products and in tests of new pharmacological treatments. Therefore, predictive and diagnostic genetic testing for these highly penetrant mutations such as PS1 or PS2 may be appropriate for adults from families with a clear autosomal dominant pattern or inheritance, particularly those with a family history of early onset of symptoms. Testing is an option that could be discussed and that could reasonably be accepted or declined by the patients. However, the application of the ApoE test raises concerns, because although the E4 allele is associated with an increased risk of $\mathrm{AD}$, its predictive value for individuals is quite limited. [11] The small increase in diagnostic confidence provided by ApoE genotyping does not justify the burdens of testing; such testing may have value in $\mathrm{AD}$ research, but its widespread clinical use is premature until practical benefits outweigh its costs.

\section{Information technology-Alzheimer's disease}

Information technology role on Alzheimer's disease has already begun. In 2003, Intel entered in to a consortium with the Alzheimer's association, granting $\$ 1$ million in Information technology research to be directed towards AD patients. Technology such as sensor networks is being used to study the habits of Alzheimer's disease patient behavior in hopes of finding ways to learn more about $\mathrm{AD}$ and to make it more livable. This is an example of how Information technology can work for AD patients. Scientist Hans Moravec has suggested that someday, entire human brains and the consciousnesses they hold will be able to be downloaded into a computer. This would certainly avoid the problem of neuronal deterioration, but it's possible that by the time, we have the technology to move minds into machines, we will know enough about $\mathrm{AD}$ to make it a livable or curable illness.

The early stage of $\mathrm{AD}$ primarily causes memory problems. At this stage a person can live independently and only requires assistance in remembering certain tasks. Cognitive prosthetics are helpful in aiding a person to remember such ISSN 0973-2063

Bioinformation 2(3): 91-95 (2007) tasks. A pager was used to help a person remember the tasks during the early stage of AD. The Pager was able to record 80 letter alphanumeric message and then display the message at scheduled time. The person was able to perform the tasks independently within a week by using the pager. The functional memory of the person had improved after using the pager for six months.

The majority of people with AD usually fit into the category of mild to moderate disease progression. At this stage it is common for patients with $\mathrm{AD}$ to move-in with a caregiver. During these stages, persons with $\mathrm{AD}$ are prone to forgetting their way home or wandering in the streets. The use of technology for people with $\mathrm{AD}$ provides hope that they can live on their own for longer period. Global Positioning System (GPS) is the technology that may prove to be very helpful in these stages. GPS is a tracking device that can be used to identify the location of the patients. A patient can wear a GPS unit and a caregiver can be notified when the patient wanders out of the designated area. Currently GPS devices used to locate Alzheimer's patients are being used with personal locater devices.

During the moderate to late stages of $\mathrm{AD}$, behavioral disturbances need for more care. At this stage, motes can be programmed to inform caregiver of all the conditions of the patient. Sensors could be placed in the person's bed to monitor weight loss. A combination of sensors placed in chairs and infrared tags detected by cameras could inform the caregiver, if the AD patient has fallen down or is sitting in the chair.

Information technology can be used to help the caregivers with the responsibilities of monitoring the AD patients as well as informing the caregivers about $\mathrm{AD}$ and answering their questions. For example, a telephone-based intervention has been used to provide help and support to caregivers. The Project is known as REACH (Resource for Enhancing Caregiver Health) used an interactive voice response (IVR) to provide support and answers to caregivers. It is necessary for the caregiver to receive the support they need in order to perform their job easily. The World Wide Web can easily solve the problem by providing knowledge and support to the caregivers. They can get the validity of online information from the online service called Alzonline.net. [12]

Healthcare providers and caregivers are responsible for constantly monitoring the patient's condition. It is not possible for many caregivers to constantly monitor the patient's health because of lack of medical knowledge or equipment. [13] An important part of care giving consists of taking the patients to the physician, this can add further stress to the care giver and affect the accuracy of physician's judgment about the patient's condition. A healthcare provider might have a better idea regarding a patient's condition if they could monitor the patient in a natural setting. A telehealth system could be used in order 


\section{Bioinformation}

\section{www.bioinformation.net}

\section{Views \& Challenges}

for the healthcare provider to monitor the patients in their home setting.

Telehealth technology can be useful for rural caregivers. They need not drive long distances. Telehealth application can be used to monitor patients at home and eliminate unnecessary travel. A number of telehealth applications have been used to monitor the patients. Information technology can play a very important role to improve the condition of the people with Alzheimer's disease. It can provide a caregiver with information and support. On the other hand it can engage the patients in many different activities to reduce the caregivers stress. A combination of telemedicine, telecommunication projects and technologies for daily living can help us with the aging of our elders the medical conditions they will face.

\section{Electronic medical records}

The main purpose of clinical care and research is to discern patterns and modify treatment according to changing parameters, be they weight, blood pressure, plasma glucose or serum lipids. Records on paper can be scanned and browsed easily but the data may not be recorded in a uniform fashion, papers may be lost, misplaced or become complex .The relevant paper records cannot easily be sorted and they cannot be accessed across different locations. [14] Electronic medical records have advantages over paper records, vz: complete and comprehensive flexibility in storage and retrieval of data, which can be used for publication, presentation and research. Computerized guidelines can provide evidence-based recommendations by allowing access to references, showing errors and sending reminders. [15] Besides, interactive telemedicine support is possible. Information laws of the country can be expected to consider EMR as a legal document. They may be electronically signed and must be permanent. An identifier must be attached to any further modifications.

\section{Bioinformatics-Biological databases}

Bioinformatics is the science of using and developing computational tools and algorithms to help in solve different biological problems. These problems include similarity searches of unknown DNA/protein sequences and the prediction of protein structure and function. Bioinformatics can be useful in finding the causative genes for Alzheimer's disease. The genome information can be obtained from existing biological databanks to analyze their structure and function. Therefore Biological databases plays important role in bioinformatics. There are around 500 public and commercial biological databases. These databases usually contain genomics and proteomics data, but databases are also used in taxonomy. The data are nucleotide sequences of genes or amino acid sequences of proteins. Furthermore information about function, structure, localization on chromosome, clinical effects of mutations as well as similarities of biological sequences can be found.
Biological databases have become an important tool in assisting scientists to understand and explain a host of biological phenomena from the structure of biomolecules and their interaction, to the whole metabolism of organisms and to understanding the evolution of species. This knowledge helps facilitate the fight against diseases, assists in the development of medications and in discovering basic relationships amongst species in the history of life. The biological knowledge of databases is usually (locally) distributed amongst many different specialized databases. This makes it difficult to ensure the consistency of information, which sometimes leads to low data quality. We can extract the genes that are causing AD from gene cards using bioinformatics.

\section{Computer applications for clinical questions}

Although prospective learning will always be important for health care professionals, it is not possible to prospectively obtain all of the knowledge required to appropriately treat all patients. Therefore, clinicians must be able to access knowledge at the point of care that answers clinical questions and provides diagnostic and therapeutic decision support. A number of available computer applications address this need. Three of these are highlighted here. Many Internet sites can provide valuable information to clinicians, and the applications can be accessed on the Internet using the websites like www.UpToDate.com, www.infopoems.com, etc.

\section{Conclusion:}

Information technology plays a vital role in identification of the genes that are causing $\mathrm{AD}$, disease management, progression and online data collection using electronic medical records for future research. The use of technology has the potential to help the patients to be more independent and reduce stress on the caregiver. The use of Information technology is might be worth pursuing if technology advances faster than a treatment or cure.

\section{Acknowledgement:}

The authors are thankful to partial financial support from IIT up gradation grants of AUCE (A).

\section{References:}

[01] P. H. George-Hyslop, Scientific American, 283: 76 (2000) [PMID: 11103462]

[02] J. Shine, et al., Nature, 270: 494 (1977) [PMID: 593368]

[03] D. E. Bassett, et al., Nature Genet., 15: 339 (1997) [PMID: 9090377]

[04] V. A. McKusick, Genomics, 45: 244 (1997) [PMID: 9463089]

[05] J. Pavia, et al., Fundament Clin Pharmacol., 12:473 (1998) [PMID: 9794144]

[06] A. M. Goate, et al., Nature, 349: 704 (1991) [PMID: 1671712]

[07] R. Sherrington, et al., Nature, 375: 754 (1995) [PMID: 7596406] 


\section{Bioinformation}

www.bioinformation.net

\section{Views \& Challenges}

[08] E. I. Rogaev, et al., Nature, 376: 775 (1995) [PMID: 7651536]

[09] X. Wang, et al., Ann Neurol., 44: 387 (1998) [PMID: 9749607]

[10] E. H. Corder, et al., Science, 261: 921 (1993) [PMID: 8346443]

[11] L. M. McConnell, et al., Nature Med., 4: 757 (1998) [PMID: 9662356]
[12] R. L. Glueckauf and J. S. Loomis, NeuroRehabilitation, 18: 135 (2003) [PMID: 12867676]

[13] K. C. Buckwalter, et al., Fam Community Health, 25: 31 [2002] [PMID: 12802140]

[14] R. Lusk and K. Herrmann, Otolaryngol Clin North Am., 31: 289 (1998) [PMID: 9518437]

[15] F. Sullivan and J. C. Wyatt, BMJ, 331: 831 (2005) [PMID:16210284]

Edited by K. Gunasekaran

Citation: Thota et al., Bioinformation 2(3): 91-95 (2007)

License statement: This is an open-access article, which permits unrestricted use, distribution, and reproduction in any medium, for non-commercial purposes, provided the original author and source are credited. 